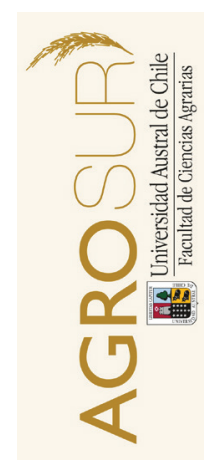

\title{
Estabilidad estructural de un Andisol (Typic Durudand) bajo bosque nativo y pradera en el Sur de Chile
}

\author{
Structural stability of an Andisol (Typic Durudand) \\ under native forest and grassland in Southern Chile \\ Zúñiga, F. ${ }^{a, b^{*}}$, Dec, D. ${ }^{a, c}$, Valle, S.R. ${ }^{a, c}$, Dörner, J. ${ }^{a, c}$, MacDonald, R. ${ }^{a \dagger}$ \\ ${ }^{a}$ Instituto de Ingeniería Agraria y Suelos, Facultad de Ciencias Agrarias, Universidad Austral de Chile, \\ Independencia 641, Valdivia, Chile. \\ ${ }^{b}$ Escuela de Graduados, Facultad de Ciencias Agrarias, Universidad Austral de Chile. \\ ${ }^{c}$ Centro de Investigación en Suelos Volcánicos (CISVo), Universidad Austral de Chile. \\ ${ }^{\dagger}$ Dedicado en Memoria del Profesor Roberto MacDonald H. (1942-2012), Instituto de Ingeniería Agraria y Suelos, \\ Facultad de Ciencias Agrarias, Universidad Austral de Chile.
}

\begin{tabular}{l} 
A R T I C L E I N F O \\
\hline Article history: \\
Received 03.06.14 \\
Accepted 19.05.15 \\
\hline Keywords: \\
Native forest \\
Grassland \\
Porosity \\
Organic matter \\
Aluminum oxalate \\
\hline Original Research Article, \\
Soil Science \\
\hline *Corresponding author: \\
Felipe Zúñiga \\
E-mail address: \\
felipezunigaugalde@gmail.com
\end{tabular}

\begin{abstract}
A B S T R A C T
The enabling of forests to achieve productive systems is one of the most significant causes of land use change in Southern Chile, and it can be evaluated through changes in soil structure. The structural stability of a volcanic soil and its relationship with the colloidal fraction under forest and grassland was evaluated in this study. Disturbed and undisturbed samples were collected for soil chemical characterization and evaluation of hydraulic and mechanical properties, aditionally qualitative properties of the organic matter were determined, for both uses, at three depths $(5,20$ and $50 \mathrm{~cm})$. For the purposes of statistical analysis land use was consider as a factor.

The land use change from grassland to forest caused: i) an increase of $38 \%$ in bulk density at $5 \mathrm{~cm}$, an increase of $44 \%$ and $75 \%$ of the organic matter content at 20 and $50 \mathrm{~cm}$; ii) an increase of $33 \%$ allophane at $5 \mathrm{~cm}$ and a reduction in the effective porosity of $14 \%$ between 5 and $20 \mathrm{~cm}$; iii) an increase of $180 \%$ in air permeability at $50 \mathrm{~cm}$, a $54 \%$ decrease in the variation of the average diameter of aggregates and a $32 \%$ decrease of the repellency index at $5 \mathrm{~cm}$.

Soil physical functioning improved after a land use change. This might be determined by the qualitative properties of soil organic matter and its interaction with the matrix, oxides and hydroxides of $\mathrm{Al}$ and Fe.
\end{abstract}

\section{RESUMEN}

La habilitación de bosques a sistemas productivos, es una de las principales causas del cambio de uso de suelo en el Sur de Chile, la que puede ser evaluada a través de cambios en la estructura del suelo. Se evaluó la estabilidad estructural de un suelo volcánico y su relación con la fracción coloidal, bajo bosque y pradera. Se colectaron muestras disturbadas y no disturbadas para caracterizar químicamente el suelo y evaluar propiedades hidráulicas y mecánicas, además de propiedades cualitativas de la materia orgánica, en ambos usos, a tres profundidades $(5,20$ y $50 \mathrm{~cm})$. Para el análisis estadístico se consideró como factor el uso del suelo.

El cambio de uso, de bosque a pradera, provocó: i) un incremento de $38 \%$ en la densidad aparente a $5 \mathrm{~cm}$, un incremento de $44 \%$ y $75 \%$ en el contenido de materia orgánica a 20 y $50 \mathrm{~cm}$; ii) un incremento del alofán en un 33\% a $5 \mathrm{~cm}$ y una reducción de la porosidad efectiva en $14 \%$ entre 5 y $20 \mathrm{~cm}$; iii) un incremento de $180 \%$ en la permeabilidad de aire a $50 \mathrm{~cm}$ y una disminución en la variación del diámetro medio de los agregados de un 54\% y del índice de repelencia en un $32 \%$ a $5 \mathrm{~cm}$.

El funcionamiento físico del suelo mejoró luego de un cambio de uso. Esto podría estar determinado por las propiedades cualitativas de la materia orgánica del suelo y su interacción con la matriz y óxidos e hidróxidos de Al y Fe.

Palabras clave: Bosque nativo, pradera, porosidad, materia orgánica, aluminio oxalato.

\section{INTRODUCCIÓN}

El cambio en el uso del suelo representa la alteración humana más importante sobre los ecosistemas terrestres (Vitousek et al., 1997), afectando los ciclos biogeoquímicos, la biodiversidad global y acelerando el cambio climático (Vitousek et al., 1997; Sala et al.,
2000; Lal, 2011). Nuestra necesidad de producción de alimentos (FAO, 2009) y vivienda han provocado profundas alteraciones en el uso del suelo (Klein y Ramankutty, 2004).

En Chile, este proceso comenzó y se desarrolló con la colonización española y alemana (Mariño de Lovera, 1865; Otero, 2006), principalmente entre las regiones 
del Maule y Los Lagos ( $35^{\circ}$ a $44^{\circ} \mathrm{LS}$ ). Lara et al. (2012), indican que de la superficie cubierta originalmente por bosque nativo entre estas regiones, ha disminuido en aproximadamente un $51 \%$ trayendo consigo efectos económicos, ambientales y sociales negativos (Nahuelhual et al., 2012), los que también se manifiestan en los suelos, particularmente en los derivados de cenizas volcánicas (Andisoles). Estos suelos representan entre el 50 y 60\% de la superficie arable (Besoain, 1985), cubriendo también un amplio rango de usos (bosques nativos, plantaciones exóticas, praderas, zonas cultivo y huertos frutales). Soil Survey Staff (2010), señala que un suelo con características ándicas debe poseer menos de $25 \%$ de carbono orgánico, una baja densidad aparente $\left(<0,9 \mathrm{~g} \mathrm{~cm}^{-3}\right)$, alta retención de fosfatos ( $\geq 85 \%$ ) y un contenido de $A l_{o}+1 / 2 F_{o} \geq 2 \%$, lo que se refleja en una alta carga variable, asociada a su mineralogía no cristalina (Shoji et al., 1993).

Guo y Gifford (2002), indican que un cambio de uso de bosque nativo a plantación reduciría el stock de $\mathrm{C}$ en el suelo en un 13\%, mientras que ocurriría un incremento de $8 \%$ en un cambio desde bosque nativo a pradera. Bajo estas condiciones de uso en Andisoles, algunos autores han evaluado el comportamiento hidráulico (Ellies et al., 1997; Dec et al., 2012) y mecánico (Ellies et al., 2000, Dörner et al., 2012), destacando la alta capacidad de conducción y retención de agua, así como su alta capacidad de resistencia. Estas propiedades son altamente dependientes de la estabilidad estructural y por consecuencia del contenido de materia orgánica del suelo (Six et al., 2000; Lal, 2004). De acuerdo al modelo de jerarquía de agregados propuesto por Tisdall y Oades (1982), los macroagregados del suelo $(\phi=>250 \mu \mathrm{m})$, serían los más afectados por una alteración en el uso del suelo, mientras que la estabilidad de los microagregados sería independiente del manejo del suelo o su condición vegetacional.

Además de las propiedades cuantitativas de materia orgánica del suelo, sus propiedades cualitativas juegan un rol clave en la estabilización estructural (Tisdall y Oades, 1982), las que se pueden evaluar a través de la repelencia de los agregados al agua. Doerr et al. (2006), sugieren que la repelencia al agua se relaciona con el tipo de vegetación presente asociada a los cambios en su uso. Ellies et al. (1996), en suelos de origen volcánico del Sur de Chile, indican que esta estabilización se favorece por la presencia de compuestos como ácidos grasos y ceras capaces de unirse a minerales de arcilla y óxidos e hidróxidos presentes en el suelo, mecanismos que han sido descritos por Sollins et al. (1996). De acuerdo al tipo de Andisol, predominarían mecanismos asociados a la formación de complejos Al-humus o a la formación de alofán (Baumgarten et al., 2013), lo que sería altamente dependiente del pH (Huygens et al., 2005), siendo estos últimos factores los que podrían estar explicando los niveles de C en estos suelos (Matus et al., 2006; Garrido y Matus, 2012).
En base a lo anterior se plantea que las alteraciones que ocurren sobre la porosidad del suelo por efecto de un cambio en su uso (de bosque nativo a pradera naturalizada mejorada) no son proporcionales con la posterior capacidad de conducción de agua y/o aire. Esto sugiere que las propiedades cuantitativas y cualitativas de la materia orgánica serían el factor principal en la estabilización estructural a nivel de macroagregados, mientras que a escala de microagregados sería la reactividad de la fracción coloidal. En ese contexto, los objetivos de este trabajo fueron: i) evaluar propiedades hidráulicas y mecánicas del suelo bajo dos usos (bosque nativo secundario y pradera naturalizada mejorada) y ii) relacionar la estabilidad estructural del suelo de dichos usos, con propiedades cualitativas y cuantitativas de la materia orgánica, así como también, con el contenido de alofán y aluminio en oxalato de un Andisol.

\section{MATERIAL Y MÉTODOS}

\section{Sitio e historial de manejo}

En la zona predomina el clima templado lluvioso con influencia mediterránea (Cfsb) (Kottek et al., 2006), temperaturas medias anuales entre 10 y $12^{\circ} \mathrm{C}$ y precipitaciones promedio de $2.000 \mathrm{~mm}$ (Subiabre y Rojas, 1994). Geomorfológicamente, el sitio se ubica en la sección oriental de la depresión intermedia a orillas del lago Ranco (Coordenadas UTM: 5554560 N, 715968 E). La elevación promedio es de $330 \mathrm{msnm}$ presentando una topografía compleja con pendientes que varían gradualmente entre 4 y 15\% (IREN-UACh, 1978). El suelo se formó a partir de cenizas volcánicas depositadas sobre morrenas basales y laterales de origen glaciar. Morfológicamente los horizontes superficiales variaron en coloración entre pardo y pardo oscuro en el matiz 10YR, lo que podría estar relacionado con los aportes orgánicos de la vegetación observada en ambos sitios (Schlatter et al., 2003). La densidad aparente (da) varió entre 0,49 a $0,71 \mathrm{~g} \mathrm{~cm}^{-3}$ y su clase textural varió entre franco a franco limoso. Estas características, asociadas al régimen de temperatura y humedad údico que caracteriza a este suelo lo clasifican como Typic Durudand (Soil Survey Staff, 2010) y pertenece a la serie Los Lagos (LLO) (CIREN, 2003).

El sitio bajo bosque nativo secundario está compuesto principalmente por Nothofagus obliqua, Aextoxicon punctatum, Nothofagus dombeyi, Amomyrtus luma, Luma apiculata, Weinmannia trichosperma y Lapageria rosea, la presencia de especies forestales tipo robleraulí-coigüe, indicarían sitios profundos y bien drenados. La pradera naturalizada se encontraba dominada por Lolium perenne, Dactylis glomerata, Holcus lanatus, y Trifolium repens, la que era utilizada para el pastoreo por bovinos de carne (Aberdeen angus). Ambos sitios poseían un tiempo de residencia mayor a los 30 años. 
Mayores antecedentes del sitio de estudio se encuentran en Dörner et al. (2011) y Baumgarten et al. (2013).

\section{Muestreo de suelos}

Se utilizó un bastón agrológico (Eijkelkamp, 1 m de longitud operacional y $0,3 \mathrm{~m}$ de diámetro), para determinar la profundidad efectiva y homogeneidad del suelo en función de sus características morfológicas como color y clase textural (Boden, 1994). Posterior a las prospecciones al azar ( $\approx 10$ por sitio) se seleccionaron áreas representativas, una en bosque y otra en pradera que poseían características morfológicas similares y pendientes entre $1-5 \%$. Por cada sitio, se excavó una calicata de $\approx 1 \mathrm{~m}^{3}$ de volumen. En cada calicata, se establecieron 3 profundidades de muestreo $(5,20$ y $50 \mathrm{~cm})$ y por cada profundidad se colectaron 10 cilindros de acero inoxidable $\left(v: 250 \mathrm{~cm}^{3}\right)$ para evaluar las propiedades hidráulicas, arrojando un total de 60 muestras de suelo no disturbadas. Adicionalmente, se recolectaron muestras de suelo disturbado para caracterizar químicamente el suelo $(\approx 5 \mathrm{~kg}$ por profundidad y sitio de muestreo) y bloques de suelo no disturbados para evaluar su estabilidad mecánica en laboratorio (Hartge y Horn, 2009). En todos los casos, las muestras de suelo fueron guardadas y transportadas en cajas plásticas selladas, para evitar daños mecánicos y pérdidas de humedad.

\section{Análisis de laboratorio}

\section{Caracterización general del suelo}

Se determinó la distribución del tamaño de partículas (Day, 1965) utilizando suelo seco $\left(\approx 25^{\circ} \mathrm{C}\right)$ tamizado a $2 \mathrm{~mm}$, se eliminó la materia orgánica y cementantes presentes en el suelo según Sandoval et al. (2012). La densidad de partículas y densidad aparente, se determinaron a través del método del pesaje sumergido y a través del método del cilindro, respectivamente (Forsythe, 1974). Se determinó el contenido de carbono orgánico utilizando el método de digestión húmeda de Walkley-Black (Sadzawka et al., 2006), se estimó indirectamente el contenido de materia orgánica, asumiendo que un $58 \%$ de $\mathrm{MO}$, corresponde a carbono orgánico. Se extrajo una fracción del aluminio reactivo del suelo $\left(A l_{e x t}\right)$ utilizando una solución de acetato de amonio a pH 4,8; posteriormente, se determinó su concentración, utilizando espectrofotometría de absorción atómica.

Mediante las extracciones de $A l_{o}$ y $S i_{o}$ utilizando como agente extractante una solución de oxalato ácido, y de pirofosfato de sodio $\left(A l_{p}\right)$ (Parfitt y Henmi, 1982), se determinó indirectamente el contenido de alofán a partir de la fórmula propuesta por Parfitt y Wilson (1985).

$$
(\alpha)=\frac{S i_{o}}{23,4-5,1\left(\frac{A l_{o}-A l_{p}}{S i_{o}}\right)}
$$

Donde:

$\alpha$ : contenido de alofán [\%]; $\mathrm{Si}_{\mathrm{o}}$ : contenido de silicio en extracto de oxalato ácido [\%]; $A l_{o}$ : contenido de aluminio en extracto de oxalato ácido [\%]; $A l_{p}$ : contenido de aluminio en extracto de pirofosfato de sodio [\%].

\section{Propiedades hidráulicas y mecánicas del suelo}

Se determinaron propiedades hidráulicas como la curva de retención de agua (Sandoval et al., 2012), conductividad hidráulica saturada (Hartge y Horn, 2009), conductividad de aire (Dörner y Horn, 2006), el índice de repelencia al agua (Hallet y Young, 1999) y mecánicas como la estabilidad de los agredados al agua (Hartge y Horn, 2009).

La conductividad hidráulica en fase saturada (Ks: $n$ $=10 ; v=250 \mathrm{~cm}^{3} ; h=5,03 \mathrm{~cm}, \phi=7,96 \mathrm{~cm}$ ) se determinó utilizando un permeámetro de carga constante (Eijkelkamp), evaluándola en los siguientes intervalos de tiempo: 1, 3, 6, 12, 24, 48, y 96 h, después de iniciado el flujo de agua.

A partir de las mismas muestras de suelo utilizadas para determinar $K s$, se determinó la curva de retención de agua $(\mathrm{pF})$. Posterior a la determinación de $K s$, las muestras fueron saturadas lentamente por $48 \mathrm{~h}$ hasta alcanzar su completa saturación $(\psi \approx 0 \mathrm{kPa})$ y luego drenadas a 6 y $33 \mathrm{kPa}$ de tensión de agua (Dörner et al., $2009 \mathrm{~b})$. Las muestras fueron secadas en horno a $105^{\circ} \mathrm{C}$ durante $24 \mathrm{~h}$ y se calculó su densidad aparente (Hartge y Horn, 2009). Para $\psi=6$ y $33 \mathrm{kPa}$, se determinó la conductividad de aire $(k)$ utilizando un flujómetro de aire con diferentes escalas (Key Instruments, Trevore, USA), para un rango de conductividad de aire de suelo entre 0,1 y $10 \mathrm{~L} \mathrm{~min}^{-1}$, aplicando una diferencia de presión máxima de 0,1 kPa (Dörner y Horn, 2006). Durante las mediciones se registraron los cambios de presión atmosférica y temperatura que fueron utilizados en los cálculos de conductividad de aire $\left(k_{l}\right)$. A partir de $k_{l}$ se determinó la permeabilidad de aire $\left(k_{a}\right)$ tal como se describe en Dörner y Dec (2007). A partir de las mismas muestras equilibradas a 6 y $33 \mathrm{kPa}$ se calculó la porosidad efectiva $(\mathrm{Pe})$, como la sumatoria de los poros de drenaje rápido (PDR) y poros de drenaje lento (PDL).

La estabilidad mecánica del suelo se determinó a través del método de tamizado en seco y en húmedo, reflejado en la variación del diámetro medio ponderado de los agregados (Vdma) (Hartge y Horn, 2009).

$$
V d m a=\frac{\sum\left(n_{i 1} * d_{i}\right)-\left(n_{i 2} * d_{i}\right)}{\sum n_{i 1}}
$$


Donde:

Vdma: variación del diámetro medio de los agregados [mm]; $n_{i 1}$ : fracción tamizado en seco [\%]; $n_{i 2}$ : fracción tamizado en húmedo [\%]; $d_{i}$ : diámetro ponderado de los agregados [mm].

A partir de las mismas muestras, utilizadas en el análisis de $K s$, se determinó un índice de repelencia al agua del suelo (sortividad) a partir del método utilizado por Hallett y Young (1999). A partir de la relación entre las tasas de infiltración de etanol y agua, se calculó indirectamente la sortividad del suelo a través de la ecuación 3 propuesta por Tillman et al. (1989); en donde el valor de 1,95 se considera como nivel crítico de repelencia o no repelencia al agua.

$$
R=1,95 *\left(\frac{S_{e}}{S_{w}}\right)
$$

Donde:

$R$ : índice de repelencia [-]; $S_{e}$ : sortividad en etanol [mm $\left.\mathrm{s}^{-1 / 2}\right]$; $S_{w}$ : sortividad en agua $\left[\mathrm{mm} \mathrm{s}^{-1 / 2}\right]$.

\section{Análisis estadístico}

Para el análisis de las variables como porosidad efectiva $(P e)$, conductividad hidráulica en fase saturada $(K s)$, permeabilidad de aire $\left(k_{a}\right)$, variación del diámetro medio de los agregados ( $V d m a$ ) e índice de repelencia $(R)$, se consideró como factor el uso del suelo (bosque $v s$. pradera). Se determinaron los supuestos estadísticos de normalidad y homogeneidad de varianza utilizando test de Shapiro-Wilks y Levene. Posteriormente, se desarrollaron análisis de varianza (ANDEVA) y cuando se presentaron diferencias estadísticamente significativas entre las medias $(p \leq 0,05)$, se realizó un test de Tukey HSD con un nivel de confianza de un 95\%. Estos análisis también fueron realizados para las características físico-químicas del suelo.

Las variables: variación del diámetro medio de los agregados vs. alofán y $A l_{o}$, y materia orgánica $v s . A l_{p}$ se evaluaron con un modelo lineal simple, donde se utilizaron el coeficiente de determinación $\left(r^{2}\right)$, el valor de significancia de la ecuación $(p)$ y el error estándar de la ecuación $(S y . x)$, como criterios de selección del modelo.

\section{RESULTADOS}

\section{Aspectos generales asociados a suelos derivados de cenizas volcánicas}

Las características físico-químicas del suelo correspondiente a la serie Los Lagos (LLO) se presentan en el Cuadro 1.
La distribución del tamaño de partículas (textura) presenta un predominio de la fracción arenosa $(\phi$ : 2000-63 $\mu \mathrm{m}$ ) bajo la condición de bosque a los 20 y 50 $\mathrm{cm}$, mientras que en superficie $(5 \mathrm{~cm})$ predomina el limo $(\phi: 63-2 \mu \mathrm{m})$. Bajo el uso de pradera, la fracción limosa predomina en todo el perfil $(5$ a $50 \mathrm{~cm})$. Respecto al contenido de arcilla, este disminuye significativamente en profundidad $(p \leq 0,05)$ bajo el sitio de bosque, tendencia que se mantiene bajo la condición de pradera, pero no de manera significativa entre profundidades de muestreo $(p>0,05)$.

Por su parte la densidad de partículas (dp) bajo el suelo de bosque incrementa significativamente $(p \leq$ 0,05 ) en un $15 \%$ a $20 \mathrm{~cm}$ y en un $25 \%$ a $50 \mathrm{~cm}$, con respecto al valor de $2,05 \mathrm{~g} \mathrm{~cm}^{-3}$ que se presenta a $5 \mathrm{~cm}$. Bajo pradera, se presenta una tendencia al incremento, la que no es significativa $(p>0,05)$.

La densidad aparente del suelo (da) bajo ambos usos, alcanza su máximo valor a $20 \mathrm{~cm}$ de profundidad, alcanzando un incremento significativo de $22 \%$ bajo la condición de bosque comparado con el valor a $5 \mathrm{~cm}$. Bajo pradera a $50 \mathrm{~cm}$, ocurre una disminución de un $23 \%$ de la densidad aparente, la que es estadísticamente significativa. Al comparar entre usos a $5 \mathrm{~cm}$ de profundidad, ocurre un incremento de un 38\% bajo el uso de pradera, alcanzando un incremento de un $18 \%$ al comparar entre usos a $20 \mathrm{~cm}$. A $50 \mathrm{~cm}$, no se presentan diferencias significativas al comparar entre usos de suelo.

El contenido de materia orgánica (MO) presenta una disminución significativa en profundidad bajo ambos usos de suelo. Utilizando como referencia el nivel de MO bajo $5 \mathrm{~cm}$, el suelo de bosque a $20 \mathrm{~cm}$ presenta una reducción del $50 \%$, y de $83 \%$ a $50 \mathrm{~cm}$. Bajo pradera, esta reducción es de un $7 \%$ a $20 \mathrm{~cm}$ y de $62 \%$ a 50 $\mathrm{cm}$. Al comparar entre usos, para una misma profundidad, se presentan diferencias significativas sólo a 20 y $50 \mathrm{~cm}$ de profundidad $(p \leq 0,05)$, incrementando el contenido de MO bajo pradera en un $44 \%$ y $75 \%$ respectivamente.

Por su parte, los niveles de alofán $(\alpha)$ incrementan significativamente en profundidad utilizando como referencia los $5 \mathrm{~cm}$ superficiales. Bajo bosque, este incremento es de un $67 \%$ a $20 \mathrm{~cm}$ y de $89 \%$ a $50 \mathrm{~cm}$; bajo pradera el incremento es de $17 \%$ y de $38 \%$ a 20 y 50 $\mathrm{cm}$. Al comparar entre usos de suelo a $5 \mathrm{~cm}$, el alofán incrementa un $33 \%$ bajo pradera $(p \leq 0,05)$, a 20 y 50 $\mathrm{cm}$ ocurre una disminución de un $6 \%$ y $3 \%$, respectivamente.

Finalmente, los niveles de aluminio extractable $\left(A l_{\text {ext }}\right)$ presentan un incremento de un $37 \%$ a $20 \mathrm{~cm}$ y una reducción de un $20 \%$ a $50 \mathrm{~cm}$, bajo bosque, cuando se utiliza como referencia la profundidad de $5 \mathrm{~cm}$; no se manifiestan diferencias en profundidad bajo pradera. Al comparar entre usos de suelo, para todas las profundidades de muestreo, los mayores niveles de $A l_{e x t}$ se alcanzan bajo pradera $(p \leq 0,05)$. 
Cuadro 1. Características físico-químicas del suelo serie Los Lagos (LLO).

Table 1. Physico-chemical properties of Los Lagos soil serie (LLO).

\begin{tabular}{|c|c|c|c|c|c|c|c|c|c|}
\hline $\begin{array}{l}\text { Sitio } \\
{[-]}\end{array}$ & $\begin{array}{l}\text { Prof. } \\
{[\mathrm{cm}]}\end{array}$ & Arena & $\begin{array}{c}\text { Limo } \\
{\left[\mathrm{g} \mathrm{kg}^{-1}\right]}\end{array}$ & Arcilla & {$\left[\mathrm{g} \mathrm{cm}^{-3}\right]$} & $\left.\mathrm{m}^{-3}\right]$ & MO & $\left.\mathrm{kg}^{-1}\right]$ & $\begin{array}{c}\boldsymbol{A l}_{\text {ext }} \\
{\left[\mathrm{mg} \mathrm{kg}^{-1}\right]}\end{array}$ \\
\hline \multirow{5}{*}{ Bosque } & 5 & $\begin{array}{c}354 \pm 8 \\
\mathrm{Ab}^{\star}\end{array}$ & $\begin{array}{c}531 \pm 14 \\
\mathrm{Ab}\end{array}$ & $\begin{array}{c}115 \pm 11 \\
\mathrm{Aa}\end{array}$ & $\begin{array}{c}2,05 \pm 0,10 \\
\mathrm{Ab}\end{array}$ & $\begin{array}{c}0,49 \pm 0,02 \\
\mathrm{Bb}\end{array}$ & $\begin{array}{c}161,5 \pm 5,6 \\
\mathrm{Aa}\end{array}$ & $\begin{array}{c}70,5 \pm 0,2 \\
\text { Bc }\end{array}$ & $\begin{array}{c}549 \pm 12 \\
\mathrm{Bb}\end{array}$ \\
\hline & \multirow{2}{*}{20} & $546 \pm 22$ & $349 \pm 30$ & $105 \pm 10$ & $2,36 \pm 0,06$ & $0,60 \pm 0,02$ & $81,5 \pm 0,8$ & $117,6 \pm 0,7$ & $743 \pm 3$ \\
\hline & & $\mathrm{Aa}$ & $\mathrm{Ba}$ & $\mathrm{Ab}$ & Aab & $\mathrm{Ba}$ & $\mathrm{Bb}$ & $\mathrm{Ab}$ & $\mathrm{Ba}$ \\
\hline & \multirow{2}{*}{50} & $502 \pm 19$ & $430 \pm 23$ & $68 \pm 5$ & $2,56 \pm 0,10$ & $0,56 \pm 0,01$ & $27,2 \pm 0,5$ & $133,1 \pm 1,2$ & $437 \pm 9$ \\
\hline & & $\mathrm{Aa}$ & $\mathrm{Ba}$ & $\mathrm{Ab}$ & $\mathrm{Aa}$ & $\mathrm{Aa}$ & $\mathrm{Bc}$ & $\mathrm{Aa}$ & $\mathrm{Bc}$ \\
\hline \multirow{6}{*}{ Pradera } & \multirow{2}{*}{5} & $377 \pm 3$ & $493 \pm 13$ & $130 \pm 14$ & $2,24 \pm 0,10$ & $0,68 \pm 0,02$ & $126,4 \pm 1,4$ & $94,0 \pm 1,6$ & $994 \pm 14$ \\
\hline & & Aa & $\mathrm{Ab}$ & $\mathrm{Aa}$ & $\mathrm{Aa}$ & $\mathrm{Aa}$ & $\mathrm{Aa}$ & Ac & $\mathrm{Aa}$ \\
\hline & \multirow{2}{*}{20} & $410 \pm 4$ & $470 \pm 9$ & $120 \pm 7$ & $2,36 \pm 0,13$ & $0,71 \pm 0,01$ & $117,5 \pm 1,2$ & $110,0 \pm 1,1$ & $947 \pm 12$ \\
\hline & & $\mathrm{Ba}$ & $\mathrm{Ab}$ & Aa & Aa & Aa & $\mathrm{Ab}$ & $\mathrm{Bb}$ & Aa \\
\hline & \multirow{2}{*}{50} & $370 \pm 16$ & $541 \pm 4$ & $89 \pm 19$ & $2,46 \pm 0,01$ & $0,55 \pm 0,01$ & $47,8 \pm 0,8$ & $129,5 \pm 0,3$ & $979 \pm 32$ \\
\hline & & $\mathrm{Ba}$ & $\mathrm{Aa}$ & $\mathrm{Aa}$ & $\mathrm{Aa}$ & $\mathrm{Ab}$ & $\mathrm{Ac}$ & $\mathrm{Ba}$ & $\mathrm{Aa}$ \\
\hline
\end{tabular}

"Arena: $\phi=2000-63 \mathrm{~mm}$, limo: $\phi=63-2 \mathrm{~mm}$, arcilla: $\phi=<2 \mathrm{~mm}$; Gran.: granular; Subang.: subangular; dp: densidad de partículas; da: densidad aparente; MO: materia orgánica; $\alpha$ : contenido de alofán; $A l_{\text {ext }}$ : aluminio extractable. Se presentan valores promedio \pm error estándar.

- A: Corresponde a la comparación entre condiciones de bosque y pradera para una misma profundidad; b: corresponde a la comparación entre profundidades bajo un mismo uso de suelo. Ambas comparaciones se realizan con un nivel de significancia de 0,05.

\section{Efecto del cambio de uso sobre el sistema poroso y su estabilidad estructural}

Los resultados indican que existe una reducción del volumen poroso al comparar el suelo de bosque vs. pradera (Fig. 1). Esta disminución de volumen $(\mathrm{Pe})$ es de un $14 \%, 13 \%$ y $4 \%$ a 5,20 y $50 \mathrm{~cm}$ de profundidad. Por su parte, los valores de $K s$, sólo presentan una disminución significativa $(p \leq 0,05)$ de un $13 \%$ a los $50 \mathrm{~cm}$ de profundidad.

La figura 2 presenta los niveles de permeabilidad de aire $\left(k_{a}\right)$ en función del uso del suelo, para tres profundidades de muestreo.

Se aprecia que bajo el suelo de pradera, ocurre un incremento significativo de un $97 \%$ y un $180 \%$ en los valores de permeabilidad de aire a 20 y $50 \mathrm{~cm}$ de profundidad. A $5 \mathrm{~cm}$, no existen diferencias entre bosque y pradera.

A pesar de que existe una disminución de porosidad efectiva en pradera -comparada con bosque- (Fig. 1), este parámetro incrementa con la profundidad bajo ambos usos de suelo, lo que se relaciona con el contenido de alofán $(\alpha)$ del suelo (Fig. 3).

Sin embargo, también se desprende que la permeabilidad de aire del suelo $\left(k_{s}\right)$, decrece en profundidad (Fig. 2) y se relaciona inversamente con un incremento en el contenido de alofán (Fig. 3).

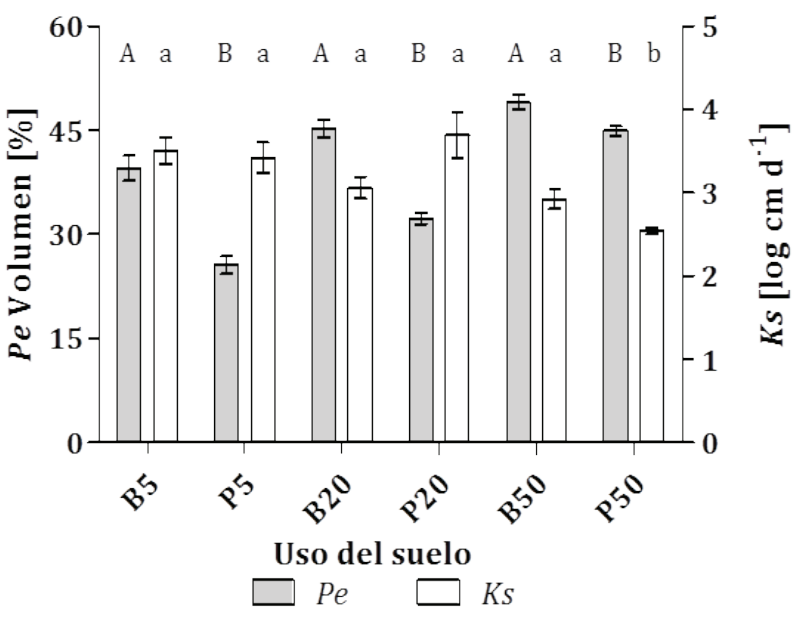

Figura 1. Porosidad efectiva $(P e)$ y conductividad hidráulica en fase saturada $(K s)$ para bosque y pradera a 5,20 y $50 \mathrm{~cm}$ de profundidad. Se presentan valores promedio \pm 1 error estándar. Letras mayúsculas indican diferencias en $P e$, mientras que letras minúsculas indican diferencias en $K S$ $(p \leq 0,05)$.

Figure 1. Effective porosity $(E p)$ and saturated hydraulic conductivity $(K s)$ for forest and grassland at 5, 20 and 50 $\mathrm{cm}$ deep. Average values are presented \pm 1 standard error. Capital letters indicate differences in $E p$, while lowercase letters indicate differences in $K s(p \leq 0.05)$. 
Bajo bosque, ocurre una disminución de Vdma en un $37 \%$ a $20 \mathrm{~cm}$ y de $66 \%$ a $50 \mathrm{~cm}$ (Fig. 4), utilizando como nivel de comparación $5 \mathrm{~cm}$ de profundidad $(p \leq 0,05)$. Al comparar entre usos de suelo, Vdma a $5 \mathrm{~cm}$ disminuye significativamente en un $47 \%$, siendo la pradera la que presenta una mayor estabilidad en sus agregados.

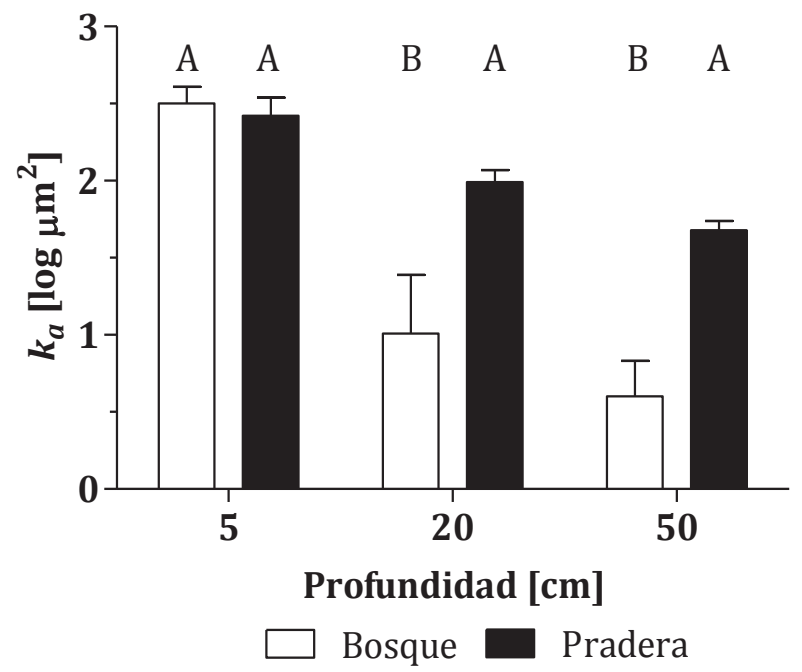

Figura 2. Permeabilidad de aire $\left(k_{a}\right)$ bajo bosque y pradera a tres profundidades $(5,20$ y $50 \mathrm{~cm})$ a $\psi=6 \mathrm{kPa}$ de tensión. Letras mayúsculas denotan diferencias entre usos a igual profundidad $(p \leq 0,05)$. Valores promedio \pm 1 error estándar $\left(k_{a}: n=9\right)$.

Figure 2. Air permeability $\left(k_{a}\right)$ under forest and grassland at three depths $(5,20$ and $50 \mathrm{~cm})$ at $\psi=6 \mathrm{kPa}$ tension. Capital letters denote differences between uses at the same depth $(p \leq 0.05)$. Mean values \pm 1 standard error $\left(k_{a}: n=9\right)$.

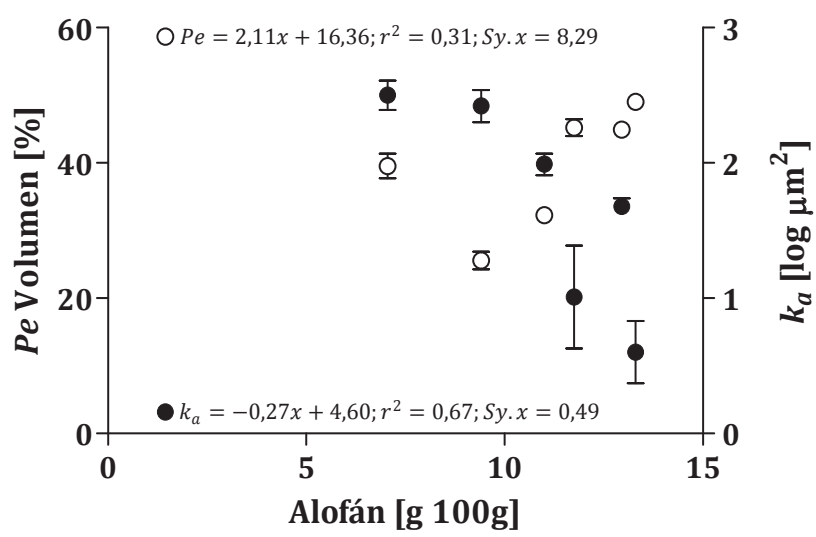

Figura 3. Relación entre la porosidad efectiva $(P e)$, permeabilidad de aire $\left(k_{a}\right)$ y el contenido de alofán $(\alpha)$. Valores promedio \pm 1 error estándar (Pe: $\left.n=10 ; k_{a}: n=9 ; \alpha: n=3\right)$.

Figure 3. Relationship between effective porosity $(E p)$, air permeability $\left(k_{a}\right)$ and allophane content $(\alpha)$. Mean values \pm 1 standard error (Ep: $n=10 ; k_{a}: n=9 ; \alpha: n=3$ ).
Relación entre la estabilidad estructural y la fracción coloidal del suelo, posterior a un cambio en su uso

Los valores del índice de repelencia $(R)$ y la estabilidad estructural se presentan en la Figura 5.

Se aprecia que el índice de repelencia al agua $(R)$ disminuye en un $32 \%, 22 \%$ y $13 \%$ a 5,20 y $50 \mathrm{~cm}$, al comparar los valores de bosque vs. pradera. Similar tendencia presenta la Vdma, al comparar ambos usos de suelo, es decir, el suelo se torna estructuralmente más estable. Para una mejor comprensión de esta información, se recomienda revisar los niveles de materia orgánica del suelo (ver Cuadro 1).

Existe una relación inversa entre la variación del diámetro medio de los agregados y los contenidos de alofán y aluminio en oxalato (Fig. 6). Si bien, alofán como $\mathrm{Al}_{o^{\prime}}$, presentan un buen ajuste del modelo $\left(r^{2}\right)$, este último, presenta un menor $p$-valor y un menor error estándar de la ecuación (Sy.x), lo que vuelve al aluminio en oxalato un mejor estimador de la estabilidad estructural comparado con el contenido de alofán.

No obstante a lo anterior, la estabilidad estructural también presenta una relación con el contenido de materia orgánica del suelo (Fig. 7), siendo esta relación di-

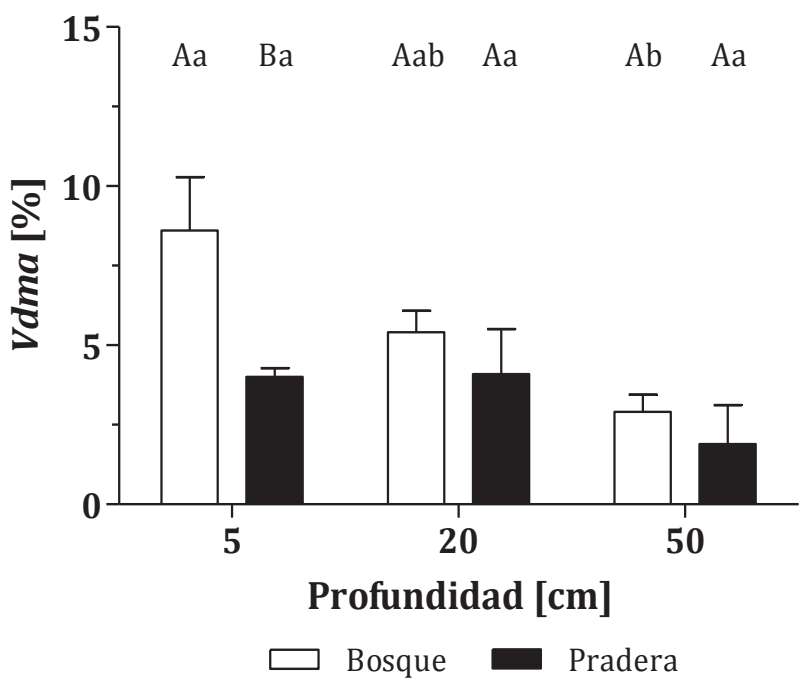

Figura 4. Variación del diámetro medio de los agregados (Vdma) para bosque y pradera según profundidad de muestreo $(5,20$ y $50 \mathrm{~cm})$. Letras mayúsculas y minúsculas distintas indican diferencias estadísticamente significativas entre los usos y profundidad, respectivamente $(p \leq 0,05)$. Valores promedio, barras indican \pm 1 error estándar $(n=3)$.

Figure 4. Mean diameter variation of aggregates $(M d v a)$ for forest and grassland according to sampling depth (5, 20 and $50 \mathrm{~cm}$ ). Different case letters indicate statistically significant differences between use and depth, respectively ( $p \leq 0.05$ ). Average values, bars indicate \pm 1 standard error $(n=3)$. 
rectamente proporcional entre ambos parámetros, es decir, a medida que el nivel de materia orgánica en el suelo es mayor, el contenido de $A l_{p}$ incrementa. Esta relación, presenta un buen ajuste del modelo $\left(r^{2}=0,75\right)$, un $p$-valor de 0,03 y un Sy.x de 28,33 que dependería del número de repeticiones $(n=3)$.

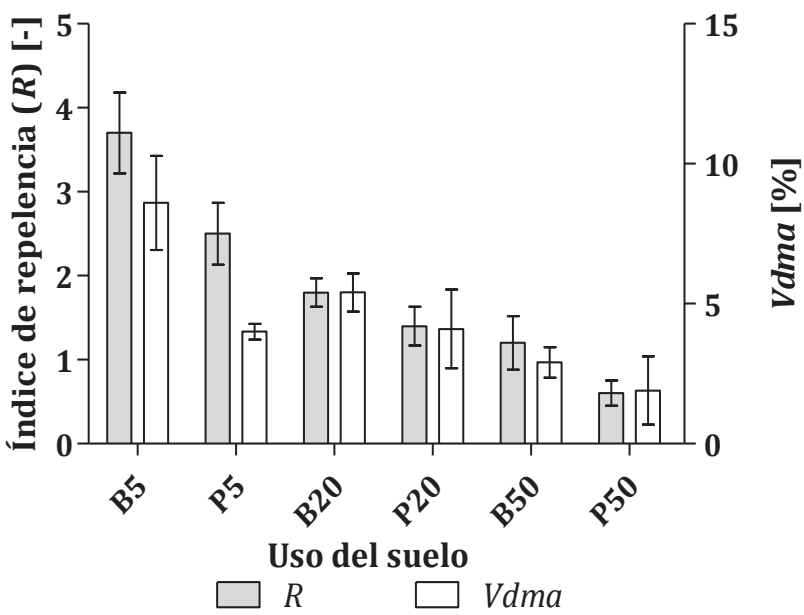

Figura 5. Índice de repelencia al agua $(R)$ y variación del diámetro medio de los agregados (Vdma) según uso de suelo y profundidad. Valores promedio \pm 1 error estándar $(R: n=10$; Vdma: $n=3$ ).

Figure 5. Water repellency index $(R)$ and mean diameter variation of aggregates (Mdva) according to land use and sampling depth. Mean values \pm 1 standard error $(R: n=10$; Mdva: $n=3$ ).

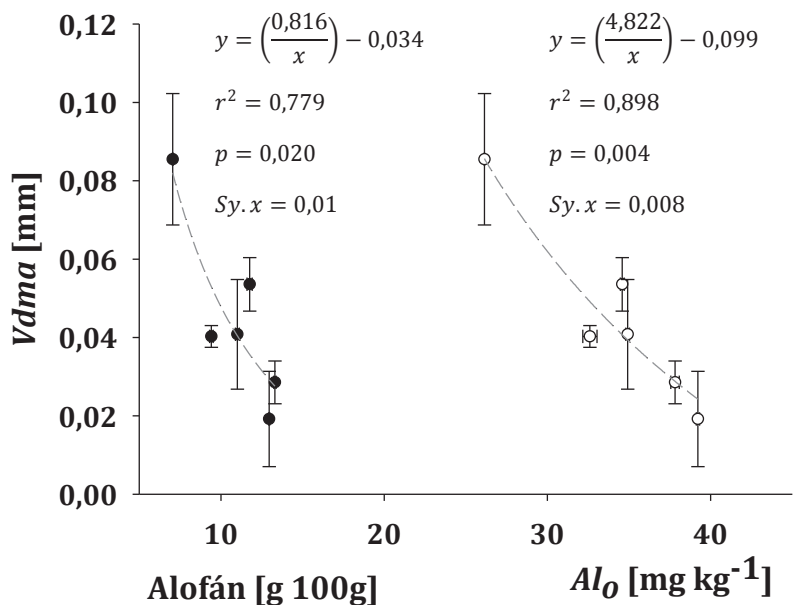

Figura 6. Relación entre la variación del diámetro medio de los agregados (Vdma) y la concentración de aluminio en oxalato $(A l)$ y contenido de alofán $(\alpha)$. Valores promedio \pm 1 error estándar. (Vdma; $\left.A l_{o} ; \alpha: n=3\right)$.

Figure 6. Relationship between mean diameter variation of aggregates $(M d v a)$ and aluminum oxalate concentration $(A l)$ and allophane content $(\alpha)$. Mean values \pm 1 standard error $(M d v a ; A l ; \alpha: n=3)$.

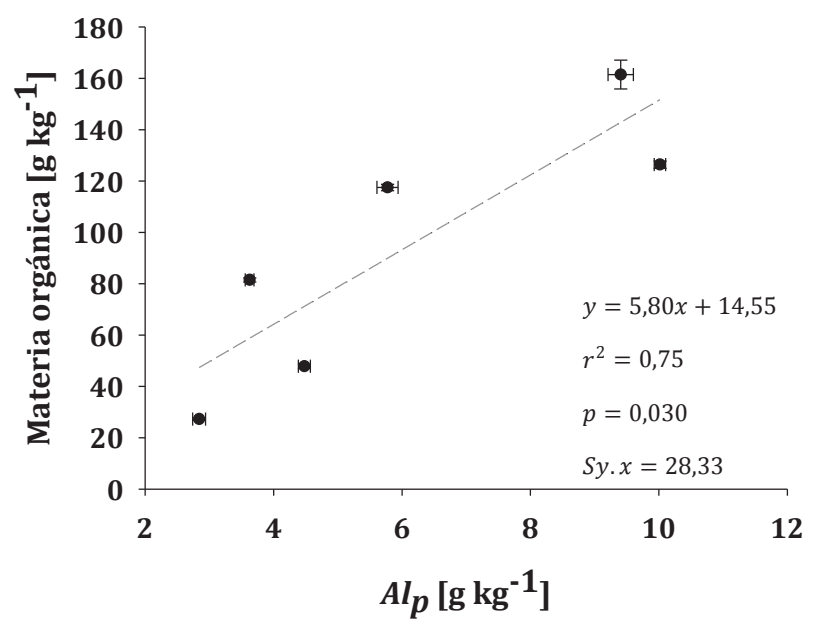

Figura 7. Relación entre el contenido de materia orgánica y la concentración de aluminio en pirofosfato $\left(A l_{p}\right)$. Valores promedio \pm 1 error estándar. (MO; $A l_{p}: n=3$ ).

Figure 7. Relationship between organic matter and aluminum pyrophosphate concentration $\left(A l_{p}\right)$. Mean values \pm 1 standard error (OM; $\left.A l_{p}: n=3\right)$.

\section{DISCUSIÓN}

Se ha documentado que los suelos derivados de cenizas volcánicas presentan propiedades particulares, como niveles de carbono orgánico menores a un $25 \%$, baja densidad aparente $\left(<0,9 \mathrm{~g} \mathrm{~cm}^{-3}\right)$, y un contenido de $\mathrm{Al}_{o}+1 / 2 \mathrm{Fe}_{o} \geq 2 \%$ (Shoji et al., 1993; Soil Survey Staff, 2010). Estas propiedades se relacionan con la formación y desarrollo del suelo y en algunos casos (p. ej. densidad aparente) pueden ser modificadas por el cambio en su uso. En base a lo anterior, propiedades "inherentes" del suelo (Karlen et al., 1997), como la textura no serían modificables por un cambio en su uso, por ello es que el predominio de la fracción arenosa en los horizontes de bosque puede ser relacionada con la meteorización de la grava encontrada bajo los $50 \mathrm{~cm}$, lo que permite un incremento de la densidad de partículas y de la densidad aparente a $20 \mathrm{~cm}$, bajo bosque. IREN-UACh (1978), señalan que resulta común encontrar gravas, guijarros y piedras en la superficie y en el perfil de suelo, por lo que las variaciones texturales encontradas en este trabajo, obedecen a la variación natural del perfil de la serie Los Lagos. Por su parte, el incremento de la fracción limosa bajo la condición pratense, podría asociarse con la posición topográfica que se encuentra la pradera (sección media de la colina) que funcionaría como una posible zona de transición o acumulación de partículas finas. Esto, sumado al constante pisoteo de los bovinos en pastoreo favorecen el asentamiento del suelo, lo que se manifiesta en un incremento en la densidad aparente en superficie 0-20 cm (Alarcón et al., 2010; Dec et 
al., 2012) lo que explicaría las diferencias entre usos. La naturaleza alofánica de este tipo de suelos y su interacción con la materia orgánica explicarían la disminución de la densidad aparente en ambos usos a $50 \mathrm{~cm}$, lo que es concordante con lo observado por Huygens et al. (2005) y Dörner et al. (2011), entre otros.

La posición topográfica del bosque (sección alta de la colina) favorece no sólo movimientos preferenciales de agua a favor de la pendiente (Dörner y Dec, 2008), sino que también flujos asociados a compuestos solubles de aluminio, lo que explicaría las diferencias entre usos, para $A l_{\text {ext }}$. Tokuchi et al. (1999), indican que la posición topográfica determinaría el tipo de horizonte orgánico (Mor, Moder, Mull) y (de acuerdo a su relación $\mathrm{C} / \mathrm{N}$ ), los procesos de transformación del $\mathrm{N}$ en el suelo, situación acorde con los niveles de materia orgánica presente en los usos de suelo evaluados.

\section{Variaciones de los indicadores de capacidad e intensidad en función del uso del suelo}

Una de las alteraciones más relevantes sobre el suelo, provocadas por la humanidad ha sido el cambio en su uso (Vitousek et al., 1997), que en el Sur de Chile, se ha concentrado en la habilitación de bosques para su uso agrícola. Este cambio de uso se puede evaluar desde una perspectiva física del suelo, que considere aspectos cuantitativos de su porosidad (como su cantidad y distribución), así como aspectos funcionales que describan la capacidad del suelo de transportar un fluido (Dörner y Dec, 2007); ambas propiedades se relacionan directamente con las variaciones que puede sufrir la estructura del suelo por un cambio en su uso (Hartge y Ellies, 1990). Estos conceptos, referidos a cantidad y funcionalidad del sistema poroso, han sido redefinidos por Horn y Kutilek (2009), como indicadores de "capacidad" e "intensidad".

Como se presenta en la Fig. 1 ocurre una disminución de la porosidad al comparar el bosque vs. pradera, no obstante, cabe preguntarse: ¿implica esta reducción, una disminución de magnitud en los parámetros que definen intensidad? El comportamiento de la conductividad hidráulica (Fig. 1), así como la permeabilidad de aire (Fig. 2) sugieren que la funcionalidad del sistema poroso es mayor bajo el suelo con cobertura pratense. En ese sentido, autores como Dörner y Dec (2007); Horn y Kutilek (2009); Berisso et al. (2012); Holthusen et al. (2012); Dörner et al. (2013), plantean que los cambios funcionales que sufriría el suelo, serían más relevantes que las variaciones cuantitativas que puedan ocurrir en el sistema poroso. El incremento en los valores de porosidad efectiva en profundidad (50 cm, Fig. 3) se asociaría con la alta reactividad y capacidad de expansión/ contracción del alofán lo que permitiría el desarrollo de una microestructura estable (Dörner et al., 2009a; Baumgarten et al., 2013), pero no tendría relación con la funcionalidad del sistema poroso. Se infiere que esta funcionalidad se relacionaría con la distribución y densidad radical de la pradera, la que presentaría una mayor acumulación de biomasa en los primeros $50 \mathrm{~cm}$ de suelo (Jackson et al., 1996). Este crecimiento radical, se puede ver favorecido por el reciclaje de nutrientes que realizan los microorganismos del suelo al degradar la biomasa proveniente de la pradera así como de los residuos de los animales en pastoreo.

Shoji et al. (1993), indican que en general los suelos derivados de cenizas volcánicas presentan excelentes propiedades físicas siendo altamente estables a nivel de macroestructura (Ellies et al., 1997) y microestructura (Velescu et al., 2010; Baumgarten et al., 2013), confiriendo a estos suelos una gran capacidad de resistir un estrés (p. ej. pisoteo animal) y de recuperarse del mismo (resiliencia). De acuerdo a Dörner et al. (2011), el suelo evaluado presenta una alta capacidad de resistir y recuperarse luego de la aplicación de estréses de tipo mecánico e hidráulico, sin embargo, esto no significa que este suelo pueda soportar un estrés indefinidamente (Dec et al., 2012); este estrés provocado por el pisoteo de los animales en pastoreo, provoca un incremento en la estabilidad estructural en la pradera (Fig. 4), particularmente a $5 \mathrm{~cm}$.

Al comparar ambos usos, el bosque presenta una menor estabilidad estructural, lo que podría estar relacionado al aporte de materia orgánica (litterfall) que no ha sufrido un proceso de descomposición; la cual si bien permite el desarrollo de espacio poroso, no participa activamente de la estabilidad de los agregados del suelo. Es posible que durante el proceso de habilitación agrícola de estos suelos, la exposición al fuego puede haber provocado un incremento en la resistencia de los agregados debido a una recristalización de óxidos e hidróxidos de Fe y Al (Mataix-Solera et al., 2011), elementos dominantes en el suelo serie Los Lagos utilizado en esta investigación.

\section{Materia orgánica, aluminio, arcilla y su influencia sobre la estabilidad estructural}

Se ha planteado que la estabilidad estructural de un suelo depende de su contenido de materia orgánica (Ellies et al., 1997; Chenu et al., 2000; Lado et al., 2004; Shrestha et al., 2007). Sin embargo, los resultados encontrados en esta investigación sugieren lo contrario, es decir, horizontes con mayores niveles de materia orgánica, se presentan menos estables, a pesar de presentar una mayor hidrofobicidad $(R<1,95$ : hidrófilo; $>1,95$ : hidrofóbo), mientras que en profundidad, el suelo se torna hidrófilo, pero más estable (ver Fig. 5, Cuadro 1).

Usos con poca intervención -como el caso de un bosque-, o con manejos conservacionistas -como la cero labranza-, favorecen la acumulación de materia orgánica (Pulleman et al., 2000), incrementando la re- 
sistencia a la humectación del suelo (Lipiec et al., 2006) comportamiento que se vincula en mayor medida con las propiedades cualitativas de la materia orgánica (Ellies et al., 1996). En ese sentido materia orgánica particulada que se encuentra en proceso de descomposición (raíces de plantas e hifas de hongos), sólo participa temporalmente en la estabilización del suelo a nivel macroestructural. El alto volumen poroso de los agregados del bosque, en conjunto con la alta gradiente hidráulica (dado que el agregado se encuentra seco al momento de ser tamizado en agua), permite que el agua ingrese con una mayor rapidez al interior de los agregados, provocando un aumento en la presión de aire, y una desintegración del agregado, lo que explicaría la menor estabilidad estructural a $5 \mathrm{~cm}$ bajo el suelo de bosque. Por su parte, componentes orgánicos aromáticos como ácidos grasos, ceras y resinas, que son más difíciles de oxidar (debido a que se encuentran protegidos físicamente), participan activamente en la estabilización del suelo a nivel microestructural (Ellies et al., 2003, 2005). Se infiere que el ingreso de residuos con una menor relación $\mathrm{C} / \mathrm{N}$ bajo pradera favorece el proceso de humificación y por consecuencia la presencia de estos compuestos orgánicos aromáticos, mejorando la estabilidad estructural del suelo.

Ellies et al. (2005), indican que una baja resistencia a la humectación, se encuentra relacionada con moléculas orgánicas de cadena corta que pueden interactuar con la matriz, óxidos e hidróxidos del suelo, lo que ayudaría a explicar la menor estabilidad bajo el suelo de bosque.

Los procesos de estabilización de la estructura pueden seguir modelos de jerarquización (Tisdall y Oades, 1982), gobernados por la materia orgánica del suelo (Six et al., 2000). Sin embargo, en suelos que poseen un predominio de carga variable (Sollins et al., 1996; Qafoku et al., 2004), la adsorción de materia orgánica y su interacción con la matriz determinarían esta estabilidad estructural, es decir conforme aumentan los niveles de $A l$ y alofán, el suelo se vuelve estructuralmente más estable. De acuerdo a lo presentado en la Fig. 6, la estabilidad de este suelo, se explicaría por los enlaces que se podrían formar entre las moléculas orgánicas y los grupos hidroxilos, tanto de las arcillas como de óxidos de Fe y Al. Yuan et al. (2000); Huygens et al. (2005), indican que estos enlaces corresponderían a atracciones electroestáticas que ocurrirían entre estos componentes, proceso que es altamente dependiente del $\mathrm{pH}$. En base a lo anterior, la estabilidad estructural de este suelo no responde al modelo de jerarquía de agregados, comportamiento documentado por Hoyos y Comerford (2005), en un Andisol colombiano. En Chile, estas relaciones (Al- $\alpha$ ) han sido evaluadas por Huygens et al. (2005), en un Typic Dystrandept "Serie Paillaco" y por Dörner et al. (2009a) en un Typic Hapludand "Serie Pelchuquín".

De acuerdo a la relación entre el contenido de materia orgánica del suelo y aluminio $\left(A l_{p^{\prime}}\right.$ Fig. 7), la forma- ción de complejos órgano-minerales estaría ocurriendo, sin embargo, participaría en menor medida en la estabilidad estructural del suelo, debido a que depende del grado de humificación de la materia orgánica, el cual a su vez es dependiente de los regímenes de temperatura y humedad del suelo (García-Rodeja et al., 2004). Matus et al. (2006) y Garrido y Matus (2012), sugieren que el contenido de alofán, $\mathrm{pH}$ al agua y $A l_{p}$, determinarían los niveles de $\mathrm{C}$ en suelos con un predominio de alofán, sin embargo, es posible que esto también se asocie a la solubilidad de las moléculas orgánicas que el suelo estaría adsorbiendo (Clunes, 2009), situación que estaría en directa relación con la formación de una microestructura estable.

\section{CONCLUSIONES}

El cambio en el uso del suelo -de bosque a pradera-, provoca un incremento en su estabilidad estructural, y una disminución en las propiedades que describen cuantitativamente el sistema poroso. Sin embargo, desde un punto de vista del funcionamiento físico de los poros, se registró un incremento de la conductividad hidráulica saturada y permeabilidad de aire bajo el suelo pratense.

La materia orgánica, juega un rol en la estabilización estructural del suelo, sin embargo las propiedades cualitativas de las moléculas orgánicas y la interacción que ocurre con la matriz del suelo y los óxidos e hidróxidos de $\mathrm{Al} \mathrm{y} \mathrm{Fe,} \mathrm{otorgan} \mathrm{al} \mathrm{suelo} \mathrm{estabilidad} \mathrm{a} \mathrm{esca-}$ la de microagregados, lo que se refleja en la capacidad de conducción de agua y aire en el suelo.

Si bien, los niveles de $\mathrm{C}$ en el suelo participan de la estabilización estructural, parece existir un componente asociado a la solubilidad del mismo, por lo que se requieren investigaciones que permitan indagar el efecto de esta solubilidad sobre la velocidad de formación de microagregados estables secuestradores de carbono, que ayuden a reducir las emisiones de $\mathrm{CO}_{2}$ desde los sistemas agrícolas y forestales.

\section{AGRADECIMIENTOS}

Este trabajo fue financiado por el proyecto FONDECYT 11060130. El primer autor, agradece la colaboración en terreno de Ivonne Orellana, Felipe Cobos y Pablo Sandoval así como los comentarios del Dr. Dante Pinochet.

\section{REFERENCIAS}

ALARCÓN, C., DÖRNER, J., DEC, D. 2010. Efecto de dos intensidades de pastoreo sobre las propiedades hidráulicas de un andisol (Duric hapludand). Agro Sur 38(1): 30-41.

BAUMGARTEN, W., DÖRNER, J., HORN, R. 2013. Microstructural development in volcanic ash soils from South Chile. Soil Tillage Research 129: 48-60. 
BERISSO, F., SCHJØNNING, P., KELLER, T., LAMANDÉ, M., ETANA, A., DE JONGE, L., IVERSEN, B., ARVIDSSON, J., FORKMAN, J. 2012. Persistent effects of subsoil compaction on pore size distribution and gas transport in a loamy soil. Soil Tillage Research 122: 42-51.

BESOAIN, E. 1985. Minerales secundarios. In: Tosso, J. (Ed.), Suelos Volcánicos de Chile. INIA, Santiago, pp. 153-214.

BODEN, A.G. 1994. Bodenkundliche kartieranleitung. 4 Auf., E. Schweizerbart'sche Verlagsbuchhandlung, Stuttgart.

CLUNES, J. 2009. Retención del aminoácido leucina por las arcillas del suelo. Tesis Ing. Agr. Universidad Austral de Chile. 48 p.

CHENU, C., LE BISSONNAIS, Y., ARROUAYS, D. 2000. Organic Matter Influence on Clay Wettability and Soil Aggregate Stability. Soil Science Society of American Journal 64: 1479-1486.

INSTITUTO NACIONAL DE INVESTIGACIÓN DE RECURSOS NATURALES (IREN), UNIVERSIDAD AUSTRAL DE CHILE (UACh). 1978. Suelos de la provincia de Valdivia. Santiago.

CENTRO DE INFORMACIÓN DE RECURSOS NATURALES (CIREN). 2003. Descripciones de suelos, materiales y símbolos. Estudio Agrológico Xa región Tomo I. Santiago.

DAY, P.R. 1965. Particle fractionation and particle size analysis. Methods of soil análisis Part I. In: Black, C.A. (Ed.), Agronomy 9: 545-567.

DEC, D., DÖRNER, J., BALOCCHI, O., LÓPEZ, I. 2012. Temporal dynamics of hydraulic and mechanical properties of an Andosol under grazing. Soil Tillage Research 125: 44-51.

DOERR, S.H., SHAKESBY, R.A., DEKKER, L.W., RITSEMA, C.J. 2006. Occurrence, prediction and hydrological effects of water repellency amongst major soil and land-use types in a humid temperate climate. European Journal of Soil Science 57: 741-754.

DÖRNER, J., HORN, R. 2006. Anisotropy of pore functions in structured Stagnic Luvisols in the Weichselian moraine region in N Germany. Journal Plant Nutrition and Soil Science 169: 213-220.

DÖRNER, J., DEC, D. 2007. La permeabilidad del aire y conductividad hidráulica saturada como herramienta para la caracterización funcional de los poros del suelo. Revista de la Ciencia del Suelo y Nutrición Vegetal 7(2): 1-13.

DÖRNER, J., DEC, D. 2008. Efecto de la estructura sobre el movimiento de agua en una catena de suelos. Agro Sur 36(2): 93-100.

DÖRNER, J., DEC, D., PENG, X, HORN, R. 2009a. Change of shrinkage behavior of an Andisol in southern Chile:
Effects of land use and wetting/drying cycles. Soil Tillage Research 106: 45-53.

DÖRNER, J., SCHROEREN, V., DEC, D., HORN, R. 2009b. Effect of Land Use on Physical Properties of a Volcanic Soil in South Chile. ISTRO $18^{\text {th }}$ Triennial Conference proceeding, June 15-19. Izmir, Turkey.

DÖRNER, J., DEC, D., ZÚÑIGA, F., SANDOVAL, P., HORN, R. 2011. Effect of land use change on Andosol's pore functions and their functional resilience after mechanical and hydraulic stresses. Soil Tillage Research 115-116: 71-79.

DÖRNER, J., DEC, D., FEEST, E., VÁSQUEZ, N., DÍAZ, M. 2012. Dynamics of soil structure and pore functions of a volcanic ash soil under tillage. Soil Tillage Research 125: 52-60.

DÖRNER, J., ZÚÑIGA, F., LÓPEZ, I. 2013. Short-term effects of different pasture improvement treatments on the physical quality of an andisol. Journal of Soil Science and Plant Nutrition 13(2): 381-399.

EIJKELKAMP AGRISEARCH EQUIPMENT. 2003. Operating instruction. Laboratory permeameters. Geisbeek, Netherlands.

ELLIES, A. 1988. Mechanical consolidation in volcanic ash soils. In: Drescher, J., Horn, R., De Boodt, M. (Eds.), Impact of Water and External Forces on Soil Structure, Catena Verlag, Supplement 11, Cremlingen, W. Germany, pp. 87-92.

ELLIES, A., GREZ, R., RAMÍREZ, C. 1996. Efecto de la materia orgánica sobre la capacidad de humectación y las propiedades estructurales de algunos suelos de la zona centro sur de Chile. Agro Sur 24(1): 48-58.

ELLIES, A., GREZ, R., RAMÍREZ, C. 1997. La conductividad hidráulica en fase saturada como herramienta para el diagnostico de la estructura del suelo. Agro Sur 25(1): 51-56.

ELLIES, A., HORN, R., SMITH, R. 2000. Effect of management of a volcanic ash soil on structural properties. International Agrophysics 14: 377-384.

ELLIES, A., RAMÍREZ, C., MACDONALD, R. 2003. Wetting capacity distribution in aggregates from soils with a different management. Journal of Food Agriculture and Environment 1(2): 229-233.

ELLIES, A., RAMÍREZ, C., MACDONALD, R. 2005. Organic matter and wetting capacity distribution in aggregates of Chilean soils. Catena 59: 69-78.

FOOD AND AGRICULTURE ORGANIZATION OF THE UNITED NATIONS (FAO). 2009. The State of Food Insecurity in the World, Economic crisis - impacts and lessons learned. FAO, Rome. 
FORSYTHE, W. 1974. Física de suelos: manual de laboratorio. IICA, San José.

GARCÍA-RODEJA, E., NÓVOA, J.C., PONTEVEDRA, X., MARTÍNEZ-CORTIZAS, A., BUURMAN, P. 2004. Aluminium fractionation of European volcanic soils by selective dissolution techniques. Catena 56: 155-183.

GARRIDO, E., MATUS, F. 2012. Are organo-mineral complexes and allophane content determinant factors for the carbon level in Chilean volcanic soils? Catena 92: 106-112.

GUO, L., GIFFORD, R. 2002. Soil carbon stocks and land use change: a meta analysis. Global Change Biology 8: 345360.

HALLET, P., YOUNG, M. 1999. Changes to water repellence of soil aggregates caused by substrate-induced microbial activity. European Journal of Soil Science 50: 35-40.

HARTGE, K., ELLIES, A. 1990. Changes in soil structure as causes by land use after clearing virgin forest. Zeitschrift für Pflanzenernährung und Bodenkunde 153: 389-393.

HARTGE, R., HORN, R. 2009. Die physikalische Untersuchung von Bóden. Praxis Messmethoden Auswertung. 4. vollst. Überarbeitete Auflage. Schweizerbart Vorlage, Stuttgart.

HOLTHUSEN, D., JÄNICKE, M., PETH, S., HORN, R. 2012. Physical properties of a Luvisol for different long-term fertilization treatments: I. Mesoscale capacity and intensity parameters. Journal of Plant Nutrition and Soil Science 175: 4-13.

HORN, R., KUTILEK, M. 2009. The intensity-capacity concept-How far is it possible to predict intensity values with capacity parameters. Soil Tillage Research 103: 1-3.

HOYOS, N., COMERFORD, N. 2005. Land use and landscape effects on aggregate stability and total carbon of Andisols from the Colombian Andes. Geoderma 129: 268-278.

HUYGENS, D., BOECKX, P., VAN CLEEMPUT, O., OYARZÚN, C., GODOY, R. 2005. Aggregate and soil organic carbon dynamics in South Chilean Andisols. Biogeoscience 2: 159174.

JACKSON, R.B., CANADEL, J., EHLERINGER, J.R., MOONEY, H.A., SALA, O.E., SCHULZE, E.D. 1996. A global analysis of root distributions for terrestrial biomes. Oecologia 180: 389-411.

KARLEN, D.L., MAUSBACH, M.J., DORAN, J.W., CLINE, R.G., HARRIS, R.F., SCHUMAN, G.E., 1997. Soil quality: a concept, definition and framework for evaluation. Soil Science Society of American Journal 61: 4-10.

KLEIN, K., RAMANKUTTY, N. 2004. Land use changes during the past 300 years. In: Klein, K., Ramankutty, N. (Eds.), Land Use, Land Cover and Soil Sciences - Vol. I UNESCOEOLSS, pp. 1-22.
KOTTEK, M., GRIESER, J., BECK, C., RUDOLF, B., RUBEL, F. 2006. World Map of the Köppen-Geiger climate classification updated. Meteorologische Zeitschrif 15: 259-263.

LADO, M., PAZ, A., BEN-HUR, M. 2004. Organic matter and aggregate-size interactions in saturated hydraulic conductivity. Soil Science Society of American Journal 68: 234-242.

LAL, R. 2004. Soil carbon sequestration to mitigate climate change. Geoderma 123: 1-22.

LAL, R. 2011. Sequestering carbon in soils of agro-ecosystems. Food Policy 36: S33-S39.

LARA, A., SOLARI, M., PRIETO, M., PEÑA, M. 2012. Reconstrucción de la cobertura de la vegetación y uso del suelo hacia 1550 y sus cambios a 2007 en la ecorregión de los bosques valdivianos lluviosos de Chile $\left(35^{\circ}-43^{\circ} 30^{\prime} \mathrm{S}\right)$. Bosque 33(1): 13-23.

LIPIEC, J., KUŚ, J., NOSALEWICZ, A., TURSKI, M. 2006. Tillage system effects on stability and sorptivity of soil aggregates. International Agrophysics 20: 189-193.

MARIÑO DE LOVERA, P. 1865. Crónica del reino de Chile. El Ferrocarril, Santiago.

MATAIX-SOLERA, J., CERDÀ, A., ARCENEGUI, V., JORDÁN, A., ZAVALA, L.M. 2011. Fire effects on soil aggregation: A review. Earth-Science Reviews 109: 44-60.

MATUS, F., AMIGO, X., KRISTIANSEN, S. 2006. Aluminium stabilization controls organic carbon levels in Chilean volcanic soils. Geoderma 132: 158-168.

NAHUELHUAL, L., CARMONA, A., LARA, A., ECHEVERRÍA, C., GONZÁLEZ, M. 2012. Land-cover change to forest plantations: Proximate causes and implications for the landscape in south-central Chile. Landscape and Urban Planning 107: 12-20.

OTERO, L. 2006. La huella del fuego: historia de los bosques nativos poblamiento y cambios en el paisaje del sur de Chile. Pehuén, Santiago.

PARFITT, R, HENMI, T. 1982. Comparison of an oxalate-extraction method and an infrared spectroscopic method for determining allophane in soil clays. Soil Science and Plant Nutrition 28(2): 183-190.

PARFITT, R., WILSON, A. 1985. Estimation of allophane and halloysite in three sequences of volcanic soils, New Zealand. In: Caldas, E.F., Yaalon, D.H (Eds.), Volcanic Soils, Weathering and Landscape Relationships of Soils on Thepra and Basalt. Catena Verlag Supplement 7, Cremlingen W. Germany, pp. 1-8.

PULLEMAN, M., BOUMA, J., VAN ESSEN, E., MEIJLES, E. 2000. Soil Organic Matter Content as a Function of Different Land Use History. Soil Science Society of American Jour- 
nal 64: 689-693.

QAFOKU, N.P., VAN RANST, E., NOBLE, A., BAERT, G. 2004. Variable charge soils: Their mineralogy, chemistry and management. Advances in Agronomy 84: 159-215.

SADZAWKA, A., CARRASCO, M., GREZ, R., MORA, M., FLORES, H., NEAMAN, A. 2006. Métodos de análisis recomendados para los suelos de Chile. Centro de Investigación Regional La Platina. Serie actas INIA 34(1), Santiago.

SALA, O., STUART, F., ARMESTO, J., BERLOW, E., BLOOMFIELD, J., DIRZO, R., HUBER-SANWALD, E., HUENNEKE, L., JACKSON, R., KINZIG, A., LEEMANS, R., LODGE, D., MOONEY, H., OESTERHELD, M., LEROY, N., SKYES, M., WALKER, B., WALKER, M., WALL, D. 2000. Global Biodiversity Scenarios for the Year 2100. Science 287: 1770-1774.

SANDOVAL, M., DÖRNER, J., SEGUEL, O., CUEVAS, J., RIVERA, D. 2012. Métodos de análisis físicos de suelos. Universidad de Concepción. Departamento de Suelos y Recursos Naturales, Número 5, Chillán.

SCHLATTER, J., GREZ, R., GERDING, V. 2003. Manual para el reconocimiento de Suelos. $3^{\text {a }}$ Ed. Universidad Austral de Chile, Valdivia.

SHRESTHA, B.M., SINGH, B.R., SITAULA, B.K., LAL, R., BAJRACHARYA, R.M. 2007. Soil Aggregate-and ParticleAssociated Organic Carbon under Different Land Uses in Nepal. Soil Science Society of American Journal 71: 1194-1203.

SHOJI, S., NANZYO, M., DAHLGREN, R. 1993. Volcanic Ash Soils. Genesis, properties and utilization. Elsevier, Amsterdam.

SIX, J., PAUSTIAN, K., ELLIOTT, E., COMBRINK, C. 2000. Soil structure and Organic Matter: I. Distribution of Aggregate-Size Classes and Aggregate-Associated Carbon. Soil Science Society of American Journal 64: 681-689.

SOIL SURVEY STAFF. 2010. Keys to Soil Taxonomy, $11^{\text {th }}$ ed. Agric. Handbk., 346. NRCS, Washington DC.

SOLLINS, P., HOMANN, P., CALDWELL, B. 1996. Stabilization and destabilization of soil organic matter: mechanisms and controls. Geoderma 74: 65-105.

SUBIABRE, A., ROJAS, C. 1994. Geografía física de la región de los Lagos. Universidad Austral de Chile, Valdivia.

TILLMAN, R., SCOTTER, D., WALLIS, M. 1989. Water-repellency and its Measurement by using Intrinsic Sorptivity. Australian Journal of Soil Research 27: 37-44.

TISDALL, J.M., OADES, J.M. 1982 Organic matter and waterstable aggregates in soils. Journal of Soil Science 33: 141-163.

TOKUCHI, N., TAKEDA, H., YOSHIDA, K., IWATSUBO, G. 1999. Topographical variations in a plant-soil system along a slope on Mt Ryuoh, Japan. Ecological Research 14: 361-369.

VELESCU, A., MEßMER, T., SCHOLTEN, T., KÜHN, P. 2010. Removal of short-range-order minerals prior to grain-size analysis of volcanic ash soils. Journal of Plant Nutrition and Soil Science 173: 799-804.

VITOUSEK, P., MOONEY, H., LUBCHENCO, J., MELILLO, J. 1997. Human Domination of Earth's Ecosystems. Science 277: 494-499.

YUAN, G., THENG, K., PARFITT, R., PERCIVAL, H. 2000. Interactions of allophane with humic acid and cations. European Journal of Soil Science 51: 35-41. 